\title{
Evolved and evolving allosteric regulation in the biosynthesis of aromatic amino acids \\ Emily Parker ${ }^{1}$ \\ ${ }^{1}$ Victoria University of Wellington, Ferrier Research Institute \\ emily.parker@vuw.ac.nz
}

Protein conformational changes and dynamics play critical roles in delivering complex enzyme function. We have examined the roles of subtle communication networks and conformational adjustments in delivering both catalysis and allosteric functionality to enzymes that play key roles in amino acid biosynthesis, allowing us to engineer enzymes with altered allosteric functionality.

Our studies have examined the complex and varied allosteric function of key enzymes involved in the biosynthesis of aromatic amino acids. 3-Deoxy-D-arabino heptulosonate 7-phosphate synthase catalyses the first step in the pathways to Trp, Phe and Tyr and shows remarkable variation in the allosteric machinery acquired for complex regulation of catalytic activity via allostery. Similarly, distinct allosteric solutions have been found for allostery in ATP phosphoribosyltransferase, which commences the pathway to His. Our work has explored the molecular mechanisms and the evolution of allostery in these two key biosynthetic pathways. 